\title{
Superconductors in the Field of Communication and Study Various Electric Properties
}

\author{
Dr. Sunder Kumar Kolli ${ }^{1}$, Sukumar Velpula ${ }^{2}$, Kiran Kumar Yenubari ${ }^{3}$ \\ ${ }^{1}$ Department of Chemistry, Annamacharya Institute of Technology \& Sciences, Hyderabad \\ ${ }^{2}$ Department of Physics, Arjun College of Technology \& Sciences, Hyderabad \\ ${ }^{3}$ Department of Physics, Sreyas Institute of Engineering \& Technology, Hyderabad
}

\begin{abstract}
Zero resistance and high current density have a major impact on electric power transmission and also enable much smaller or more powerful magnets for motors, generators, energy storage, medical equipment and industrial separations. Low resistance at high frequencies and extremely low signal dispersion are key aspects in microwave components, communications technology and several military applications. Low resistance at higher frequencies also reduces substantially the challenges inherent to miniaturization brought about by resistive, or $I^{2} R$, heating. The high sensitivity of superconductors to magnetic field provides a unique sensing capability, in many cases 1000x superior to today's best conventional measurement technology. Superconductors in the field of communication and study of various the electric properties such as Conductors, Semiconductors, Insulator and Superconductors. Superconductors specifications graphs in communication feature scope-Superconductor in the field of communication towers such that loss of electrical radiation to protect our environment.
\end{abstract}

Keywords: Zero resistance, Superconductor, Magnetic field and Intermetallic compounds

\section{Introduction}

In 1911, H. K. Onnes, a Dutch physicist, discovered superconductivity by cooling mercury metal to extremely low temperature and observing that the metal exhibited zero resistance to electric current. Prior to 1973 many other metals and metal alloys were found to be superconductors at temperatures below 23.2K. These became known as Low Temperature Superconductor (LTS) materials. Since the 1960s a Niobium-Titanium (Ni-Ti) alloy has been the material of choice for commercial superconducting magnets. More recently, a brittle Niobium-Tin intermetallic material has emerged as an excellent alternative to achieve even higher magnetic field strength. ${ }^{1}$ In 1986, J. G. Bednorz and K. A. Müller discovered oxide based ceramic materials that demonstrated superconducting properties as high as $35 \mathrm{~K}$. This was quickly followed in early 1997 by the announcement by $\mathrm{C}$. W. Chu of a cuprate superconductor functioning above $77 \mathrm{~K}$, the boiling point of liquid nitrogen. Since then, extensive research worldwide has uncovered many more oxide based superconductors with potential manufacturability benefits and critical temperatures as high as $135 \mathrm{~K}$. A superconducting material with a critical temperature above $23.2 \mathrm{~K}$ is known as a High Temperature Superconductor (HTS), despite the continuing need for cryogenic refrigeration for any application.

Arising the scope of studying superconductor in the field of communication first discuss about superconductivity of different materials. Super conductors cope in transmission of signals in terms of electromagnetic effects such as meissner effect, ac josphen, dc josphen effect, zero resistance. The experiment carried about past decades for the development of communication transmission and receiving of signals of superconducting probes or materials. Transmission of signal such as antennas, radar, transmitter and receiver to travel signals for longer distance. Building block of superconducting materials in satellite communication in ISRO, NASA etc. growth and development of superconducting materials in satellite, electromagnetic waves in the field of communication.

\section{Unique Properties}

- Zero resistance to direct current

- Extremely high current carrying density

- Extremely low resistance at high frequencies

- Extremely low signal dispersion

- High sensitivity to magnetic field

- Exclusion of externally applied magnetic field

- Rapid single flux quantum transfer

- Close to speed of light signal transmission

\section{Challenges}

- Cost

- Refrigeration

- Reliability

- Acceptance

Transportation: The rapid and efficient movement of people and goods, by land and by sea, poses important logistical, environmental, land use and other challenges. Superconductors are enabling a new generation of transport technologies including ship propulsion systems, Industry: magnetically levitated trains and railway traction transformers.

Medicine: Advances in HTS promise more compact and less costly Magnetic Resonance Imaging (MRI) systems with superior imaging capabilities. In addition, MagnetoEncephalography (MEG), Magnetic Source Imaging (MSI) and Magneto-Cardiology (MCG) enable non-invasive diagnosis of brain and heart functionality. 


\section{International Journal of Science and Research (IJSR) \\ ISSN (Online): 2319-7064}

Index Copernicus Value (2015): 78.96 | Impact Factor (2015): 6.391

Large motors rated at $1000 \mathrm{HP}$ and above consume $25 \%$ of all electricity generated in the United States. They offer a prime target for the use of HTS in substantially reducing electrical losses. Powerful magnets for water remediation, materials purification and industrial processing are also in the demonstration stages.

Communications: Over the past decade, HTS filters have come into widespread use in cellular communications systems. They enhance signal-to-noise ratios, enabling reliable service with fewer, more widely-spaced cell towers. As the world moves from analog to all digital communications, LTS chips offer dramatic performance improvements in many commercial and military applications.

Scientific Research: Using superconducting materials, today's leading-edge scientific research facilities are pushing the frontiers of human knowledge and pursuing breakthroughs that could lead to new techniques ranging from the clean, abundant energy from nuclear fusion to computing at speeds much faster than the theoretical limit of silicon technology. Engineering materials are important in everyday life because of their versatile structural properties. Other than these properties, they doplayan important role because of their physical properties. Prime physical properties of materials include electrical properties, thermal properties, magnetic properties and optical properties. The electrical behaviors of engineering materials are diverse, and so are their uses in electrical applications.

\section{Results and Discussions}

\section{Classification -Electrical conductivity}

Conductivity of solid engineering materials observed to vary over 27 orders of power. Based on their conductivity,

materials are classified as: conductors, semiconductors, and insulators.

Conductors $>10^{7}(\Omega-\mathrm{m})^{-1}$

Semiconductors $10^{-6}-10^{4}(\Omega-\mathrm{m})^{-1}$

Insulators $<10^{-10}(\Omega-\mathrm{m})^{-1}$

\section{Genesis of electrical conduction}

It is known that electrons are prime charge carriers. Electrons in metals are arranged into shells and subshells in accordance with Pauli exclusion principle with distinct energy state level. In metals many electrons will have same energy state, leading to formation of energy bands. The electrons not involved in bonding remain in what is called the core band, where as the valence electrons that form the electron gas enter into the valence band. The remaining unfilled orbitals form higher energy bands, called conduction band. It is the conduction band that gives metals and alloys the ability to freely conduct electrons. ${ }^{2}$

\section{Energy band structures}

The energy corresponding to the highest filled state at 0 Kelvin is called the Fermi energy, Ef.

Four types of band structures are possible at $0 \mathrm{~K}$ as shown in the following figure.

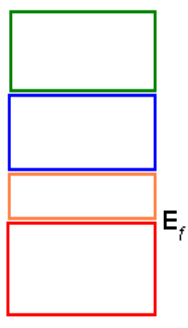

(1)

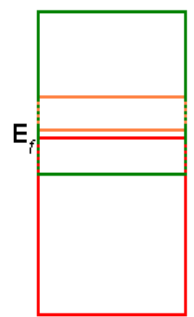

(2)

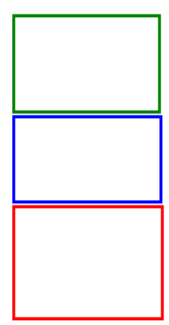

(3)

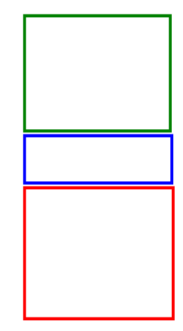

(4)
Filled (valence) band

Band gap

Empty states

Empty (conduction) band

\section{Energy band structures}

Band structures (1) and (2) are found in metals / conductors. Band structures (3) and (4) are distinguished by the size of energy band gap. Narrower energy band gap i e. size $<2 \mathrm{eV}$, is found in semiconductors, while the broad energy band gap i.e. size $>4 \mathrm{eV}$, is found in insulators. Fermi energy for the last two band structures lies within the band gap near its center. Metals have high conductivities because of the large number of free electrons that have been excited into empty states above the Fermi energy.

\section{Mathiessens rule - Resistivity}

Lattice vibrations and phonon scattering play a role in disrupting the mean free path of electrons. In addition, crystal line defects and impurity atom affect the conductivity. These scattering mechanisms act independently on one another. Thus the effective resistivity of metals can be represented as follows. With increase of temperature, thermal vibrations increase so the resistivity and vice versa. In the same manner, with increase of either defects or impurities, resistivity increases. For pure metals, the resistivity approaches zero at absolute zero temperature.

\section{Superconductivity}

Some metals lose all resistivity abruptly and completely at some low temperatures, above 0 Kelvin-phenomenon are called superconductivity and the materials that exhibit it are called superconductors. The temperature at which the resistivity vanishes is called the critical transition temperature, Tc. Many metals, solid- solution alloys, some ceramics and intermetallic compounds exhibit superconductivity. Ex.: Ti, V, Zn, W, Al, Hg, NbTi, Nb3Sn, MgB2, La-Sr-Cuoxide, YBa2Cu3O7-x, carbonnanotubes, etc. BCS (Bardeen, Cooper and Schrieffer) theory explains that the superconductivity is caused by electron- lattice interaction and that the superconducting electrons consist of paired ordinary electrons called a Cooperpair.

\section{Superconductivity: Applications in Wireless Communications}

Superconductors offer the unique advantages of ultra-low dissipation and distortion as well as intrinsic (quantum) accuracy. These advantages combine to enable what researchers with the US Army have called the "most significant change to satellite communications worldwide in 30 years." Advanced filters are already deployed in commercial wireless base stations, enabling wider range and fewer dropped calls. A more significant development involves a complete transition to all-digital receivers and 


\section{International Journal of Science and Research (IJSR) \\ ISSN (Online): 2319-7064}

Index Copernicus Value (2015): 78.96 | Impact Factor (2015): 6.391

transmitters, a migration currently in the prototype phase which promises dramatic improvements in efficiency and cost for both military and commercial wireless communication systems.

\section{All-Digital Receivers}

While HTS filters have demonstrated the unique positive impact of superconductors on the analog (non-digital) elements in wireless communications, the true revolution under development is one that takes advantage of the intrinsic linearity and quantum accuracy of superconductors to produce the world's best analog-to-digital converters. ${ }^{3-5}$ Much like digital CDs and digital television provide a superior experience as well as improved efficiency, alldigital receivers carry the same benefits to wireless communications. The crux of the improvement is in the unique ability of superconducting analog-to-digital converters to digitize a wide band of signal without the need for analog pre-processing. As a result, significant portions of the system, which usually add weight, volume, cost and distortion, are completely eliminated. In addition, the manipulation of the digital data enables full flexibility in accommodating any protocol through the use of software, thus leading to an effectively universal system where the same hardware is adapted, by software to "translate" and decode any incoming signal from any other system. Compatibility with legacy systems as well as "futureproofing" are ensured. While this technology is not yet commercially available, its development is proceeding at an impressive pace, supported in part by the US Government and in part by commercial ventures.

"Most Significant Change to Satellite Communications Worldwide in 30 years" Recently, a demonstration of the capability of superconducting All-Digital Receivers was carried out by the US Army, in which an x-band satellite communications link was closed using such a receiver. The ability to directly digitize the $\mathrm{x}$-band RF signal was proven and led to the proclamation that the "most significant change to satellite communications worldwide in 30 years" had been achieved. The heart of the system is the superconducting integrated circuit (IC). This circuit is made similarly to semiconductor ICs, but the key material here, instead of being Silicon, is the low temperature superconductor Niobium. The chip - less than half the size of a penny contains about 11,000 Josephson junctions laid out to form superconducting Rapid Single Flux Quantum (RSFQ) circuits that move picosecond-duration magnetic pulses. After many years of research, the superconductivity community worldwide has determined that such digital applications can only be harnessed using low temperature superconductors, a fact that simplifies design and fabrication of the circuits but adds the more difficult constraint of cooling the IC to 4 degrees Kelvin (by contrast to the more easily reachable 70 degrees Kelvin for HTS materials). Nevertheless, advances in cryogenics, including recent breakthroughs in pulse-tube technology, fully provide the enabling cooling platform for packaging and deploying AllDigital Receivers. In addition, the same technology can be applied to All-Digital Transmitters (and hence, All-Digital Transceivers), resulting in similar gains in performance, cost, and efficiency.

\section{Issues and Recommendations}

\subsection{Superconductivity}

Superconducting devices are so accurate they define the "Volt," goes the saying. The true testament to the intrinsic accuracy that results from the properties of superconductors is that the metrology standard for realizing the electrical unit of "Volt" is indeed a superconducting circuit. In addition to accuracy, superconductors enable the most sensitive detectors of electromagnetic radiation and are used in scientific research both at ground-based astronomy observatories as well as in space-based NASA missions.

\subsection{Instrumentation}

The earliest applications of superconducting electronics were and continue to be, custom instruments based primarily on super sensitivity in detecting magnetic fields. These instruments are based on various designs of SQUIDs and find applications in research laboratories for physics, chemistry and materials science research as well as in field geological expeditions such as airborne detection of salt domes, a frequent indicator of potential new oil fields.

\subsection{Sensors}

Besides being ultrasensitive detectors of magnetic fields, superconductors also excel in the detection of extremely faint electromagnetic signals, for example signals originating in outer space. SQUID detectors hold the record in sensitivity and are used in many a radioastronomy observatory worldwide. The detectors are used in several modes, including as discriminators of the various frequencies of the incoming signals, as in the Radio Observatory featured in the figure, as well as in camera mode providing infrared images of astronomical objects, an example being the SCUB-2 infrared camera on the James Clerk Maxwell telescope in Hawaii.

\subsection{Standards}

It took several decades of research and international collaboration to realize that superconducting devices can be the basis for a metrology standard for the electrical unit of the Volt. This is due to a fundamental property of a key superconducting element, the "Josephson Junction," which acts a frequency-to-voltage converter. Primary voltage standard systems, based on this principle, are now in virtually every national metrology laboratory, as well as in many advanced industrial research laboratories. The systems are available commercially for generating and calibrating any static voltage up to 10 Volts with accuracy up to 5 parts per billion. Continuing research by the National Institute of Science and Technology (NIST) in the US and similar institutions worldwide is focused on developing AC-voltage standards as well as arbitrary signal generators with the precision and accuracy of the existing DC-standards. 


\subsection{Radar}

Superconductive electronics can dramatically enhance antiship missile defense radars. Emerging threats include seaskimming missiles that reflect very small fractions of the total radar signal. The challenges to the radar receivers are that they must distinguish these small echoes from the huge background clutter of waves, rain, jammers and mountains on the shore in real time. Superconductivity enables the highest dynamic range digitizers and the smallest digits are meaningful to detect the most elusive threats. High dynamic range superconductive electronics provide the most advanced technology and simplify the receiver, thereby making these life-saving sensors affordable for a wide variety of Navy ships.

\section{Conclusion}

Both military and commercial applications stand to benefit from the use of superconducting All-Digital Receivers (and Transmitters and Transceivers). The more difficult technical problems to address are in military applications, due to the much wider spectrum of frequencies, protocols, and applications involved. On the commercial front, commoditylevel cost is such a competitive factor that initially, the better performance afforded by superconducting All-Digital technology is relegated to future plans pending its further evolution into a fully developed low-cost alternative. The much needed exploitation of supercomputing All-Digital technology will be enabled, perhaps uniquely, by a dedicated Governmental support to produce and demonstrate field prototypes with full performance. These demonstrations will be the needed catalyst to engage procurement plans from the Government and to place this technology on the roadmap for commercial wireless communications companies. Besides continued support for the $R \& D$ required to advance these devices and applications, an enabling technology important in facilitating the adoption of these applications and the concomitant improvement in performance of the systems they support, is a more efficient and reliable cryocooling system that is transparent to the end user. Advances are indeed occurring in this area but at a slow pace. Acceleration of this development would be of great benefit, enhancing the scientific and industrial applications of these instruments as sensors and standards as well as benefiting superconductorbased applications in communications.

\section{References}

[1] Source at (C) 2009 CCAS : Coalition for the Commercial Application of Superconductors.

[2] Module 14 electrical properties of NPTEL.

[3] Presentations of Symposium "High Temperature Superconductors in High Frequency Fields", Cologne, October 1994 in Journal of Superconductivity, vol. 8, No. 6, 1995.

[4] Workshop "The Role of Superconducting Technologies in Wireless Communication

[5] Systems", IEEE MTT-S'96International Microwave Symposium, San Francisco, June 17-21, 1996.

[6] Knack, J. Mazierska, H. Piel, Proceedings of Asia Pacific Microwave Conference, IEEE Press, December 11-14, 2007, Bangkok, pp. 187-190, 2007. 